\title{
Presence of Fluoroquinolone mono-resistance among drug-sensitive Mycobacterium tuberculosis isolates: An alarming trend and implications
}

\author{
Rohini Sharma ${ }^{\mathrm{a}, 1}$, Binit Kumar Singh ${ }^{\mathrm{a}, 1}$, Prahlad Kumar ${ }^{\mathrm{b}}$, Ranjani Ramachandran ${ }^{\mathrm{c}}$, \\ Pankaj Jorwal ${ }^{\text {a,* }}$
}

a Department of Medicine, All India Institute of Medical Sciences, New Delhi, India
${ }^{\mathrm{b}}$ National Tuberculosis Institute, Bangalore, India

${ }^{\mathrm{c}}$ World Health Organization, New Delhi, India

\section{A R T I C L E I N F O}

\section{Keywords:}

Drug Susceptibility Testing

First-line drugs

Line probe assay

\begin{abstract}
A B S T R A C T
Background: The phenomenon of Drug Resistant Tuberculosis (DRTB) has been evolving aggressively and is posing great threat to tuberculosis control programs worldwide. But what really is fueling drug resistance in high disease burden countries apart from other well known risk factors, with this intent in mind the present study was developed and carried out to. The aim was to capture the presence of Fluoroquinolone (FQ) mono-resistance among drug-sensitive TB cases.

Methods: A total of 1280 sputum smear-positive patients were enrolled in the study and were subjected for Drug Susceptibility Testing (DST) using First-Line Drugs (FLD's; Rifampicin, Streptomycin, Isoniazid and Ethambutol) using liquid culture DST and Line Probe Assay. These samples were further subjected to second-line drugs DST (ofloxacin and kanamycin) and DNA sequencing to confirm FQ mono-resistance.

Results: The occurrence of FQ mono-resistance among FLD sensitive cases was found to be 3.2\% (35/1099). Xpert MTB/RIF assay and $r p o B$ gene sequencing were showed 100\% concordance with FLD DST. A total of 35 FQ mono-resistant isolates were further sequenced for $g y r A$, gyrB and $r r s$ genes. The gene sequencing was found to be in agreement with the DST results for 34 (3.1\%) isolates with gyrA and gyrB genes.

Conclusion: Presence of FQ resistance among drug sensitive TB cases is a red flag sign. The findings of the present study suggest that, second-line DST should be routinely performed not only for drug-resistant cases but also for drug-sensitive cases so as to capture prevailing true drug resistance at an initial stage.
\end{abstract}

\section{Introduction}

Fluoroquinolones (FQs) are a group of antibiotic that have tremendous activity against Mycobacterium tuberculosis (Mtb). They are bactericidal and enjoy a distinct status among second-line anti-tuberculosis (ATT) drugs due to their high oral bioavailability, good tolerability and low-to-moderate cost. ${ }^{1,2}$ They are being widely prescribed all over the globe, especially in low to middle income countries due to their broad spectrum of antibacterial activities which covers various infections such as pneumonia, sinusitis and urinary tract infections. ${ }^{3}$

In the last two decades, FQ mono-resistant strains of $M t b$ have emerged globally and the incidence have been increasing day by day. ${ }^{4}$ Resistance to second-line anti-TB drugs is a cause of grave concern for the community and tuberculosis control programmes. ${ }^{5}$ Mostly the patients with poor treatment outcomes are closely linked with presence of FQ resistance, this further complicates the case eventually leading to development of Extensively Drug-Resistant TB (XDR-TB). ${ }^{6}$

Among drug sensitive tuberculosis cases the Drug Susceptibility Testing (DST) for FQs is not being routinely performed and periodic surveillance of FQ resistance is being done infrequently. The presence of FQ resistance is more commonly seen from those regions where they are widely prescribed and even misused. ${ }^{7}$ World Health Organization (WHO) has taken up the issue of FQ resistance among DR-TB cases, but the exact prevalence of FQ resistance among drug sensitive TB cases is still not known. ${ }^{5}$

Globally, there is limited information available on FQ mono-resistance based on culture and DST among drug sensitive $M t b$ isolates and none is from a resource limited High Disease Burden Country

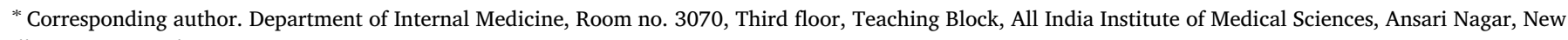
Delhi, 110 029, India.

E-mail address: pankajjorwal.aiims@gmail.com (P. Jorwal).

${ }^{1}$ Both the authors have equal contribution.
} 
(HDBC) like India. Therefore, this observational study was designed to look for presence of FQ mono-resistance among first-line drug sensitive TB cases under programmatic settings of Revised National Tuberculosis Control Programme (RNTCP) at a tertiary care center.

\section{Methods}

\subsection{Participants}

From January 2014 to December 2016, a total of 1280 patients who were sputum smear-positive pulmonary tuberculosis cases were enrolled for the study (including new and previously treated cases) at Intermediate Reference Laboratory (IRL), Department of Medicine, All India Institute of Medical Sciences, New Delhi. The study was approved by Institutional Ethics Committee and written and informed consent was obtained from all the subjects.

\subsection{Tests performed}

All the sputum samples were subjected to Ziehl-Neelsen (ZN) staining, liquid culture inoculation, Line probe assay (LPA) and DST for first-line and second-line drugs using liquid culture techniques. Gene sequencing was performed on all the FQ resistant strains.

\subsection{Specimen processing}

All sputum specimens were processed by the standard decontamination protocol (NALC-NAOH method). The processed sample was then used for culture inoculation in BACTEC Mycobacterium Growth Indicator Tube (MGIT) for liquid culture. ${ }^{8}$

\subsection{Acid-fast bacilli (AFB) smear}

One slide for AFB was made directly from each sample; stained using ZN staining method as per the standard protocol and observed under the microscope. ${ }^{9}$

\subsection{MGIT 960 liquid culture}

Processed sputum specimens were inoculated into the liquid culture using non-radiometric automated MGIT-960 isolation system [Becton Dickinson, Sparks, MD, USA]. Positive cultures were further subjected to immunochromatographic assay (ICA) kit (SD MPT64TB Ag kit developed by Standard Diagnostics, South Korea) for the differentiation of $M t b$ complex and non- $M t b$ complex.

\subsection{Drug susceptibility testing [DST]}

DST was performed using BACTEC MGIT 960 system by 1\% proportion method for both first-line [streptomycin (SM), isoniazid (INH), rifampicin (RIF) and ethambutol (EMB)] and second-line [ofloxacin (OFX) and kanamycin (KM)] anti-tuberculosis drugs (ATT) as per the standard operating procedure according to the manufacturer's instructions. $^{8}$

\subsection{Line probe assay [LPA]}

The Genotype MTBDR plus LPA version 2.0 was performed on all sputum smear-positive specimens, according to the manufacturer's (Hain Lifescience, Nehren, Germany) instructions. ${ }^{10}$

\subsection{Genetic sequencing}

DNA sequencing was carried out with $\mathrm{ABI}$ prism 3130xl genetic analyzer (Applied Biosystem). The genomic bacterial DNA extraction of culture isolates was done by heat lysis method. ${ }^{11}$ The hot-spot regions of $r p o B$, gyrA, gyrB and rrs genes were amplified by polymerase chain reaction (PCR) and DNA sequencing was done with specific primers. ${ }^{12,13}$ PCR product was examined by gel electrophoresis in $2 \%$ agarose gel. Cycle sequencing was carried out using Big Dye Terminator v3.1 cycle sequencing kit (Applied Biosystem).

\subsection{Statistical analysis}

Data was analyzed using Stata 11.2 (Texas, USA) and presented as frequency (percentage) and mean (SD). Sensitivity, specificity, negative predictive value, and positive predictive value with $95 \%$ confidence intervals were calculated. The sequencing results were analyzed using Bio Edit software and alignment was done using Clustal W.

\section{Results}

\subsection{Characteristics of the study population}

A total of 1280 sputum smear positive patients were enrolled in the study with a male: female ratio of 3:1 and mean age being 35.29 \pm 15 years. Overall culture positivity rate was 96\% (1228/1280) among smear positive sputum specimens. One hundred and eighty-one samples were excluded; as eighty-seven (87/1228; 7\%) were found to MDR-TB patients (excluded as according to the inclusion criteria of the study), culture contamination (38/1228; 3.1\%), non- $M t b$ complex (04/1228; $0.3 \%$ ) and culture negatives (52/1280; 4\%). Thus, a total of $1099 \mathrm{drug}$ sensitive $M t b$ isolates were taken for the study (Fig. 1).

\subsection{Comparison of FLD results by Xpert MTB/RIF assay, LPA and rpoB genes sequencing}

Xpert MTB/RIF assay and $r p o B$ gene sequencing was done in $35 \mathrm{FQ}$ mono-resistance $M t b$ isolates in order to look for RIF resistance among the isolates. Interestingly, the results for the all thirty five FQ monoresistance isolates showed sensitive to RIF. The results were $100 \%$ concordance when it was sequences for $r p o B$ gene sequencing and no mutations were identified among thirty-five FQ resistance isolates, which were resistant by phenotypic DST for FQ and sensitive for FLDs.

\subsection{Drug resistance pattern to second-line anti-TB drugs (SLDs) in drug} sensitive Mtb isolates

All non MDR-TB (1099) isolates by phenotypic DST were subjected to second-line DST, out of which FQ mono-resistance (OFX) was found in $3.2 \%(35 / 1099)$ isolates and no incidence of XDR-TB was found.

\subsection{Mutation pattern of SLDs by DNA sequencing}

All 35 phenotypic FQ resistance isolates were then further sequenced for $g y r A$, gyrB and rrs genes. Most common single mutation in gyrA gene was D94A in 18 (51.43\%) isolates. Other mutations included D94Y in 9 isolates (25.71\%) and A90V mutation in 6 isolates (17.14\%). In gyrB gene a single type of mutation was observed at position 1496 (AAC-ACC) leading to amino acid change from N499T. Phenotypic DST and gene sequencing revealed concordance for gyrA, gyrB in 34 (3.1\%; $34 / 1099)$ isolates and in case of $r r s$ gene one $(0.1 \% ; 1 / 1099)$ isolated mutation was observed at position 1401 (CAC-CGC) (Table 1) (see Table 2).

\section{Discussion}

Despite many advances in diagnosis and management of pulmonary tuberculosis, cases of DRTB are increasing day by day. By the implementations of newer and rapid diagnostics modalities like nucleic acid amplification test (Xpert MTB/RIF) and probe hybridization techniques (line probe assay), drug resistance can be captured at an 


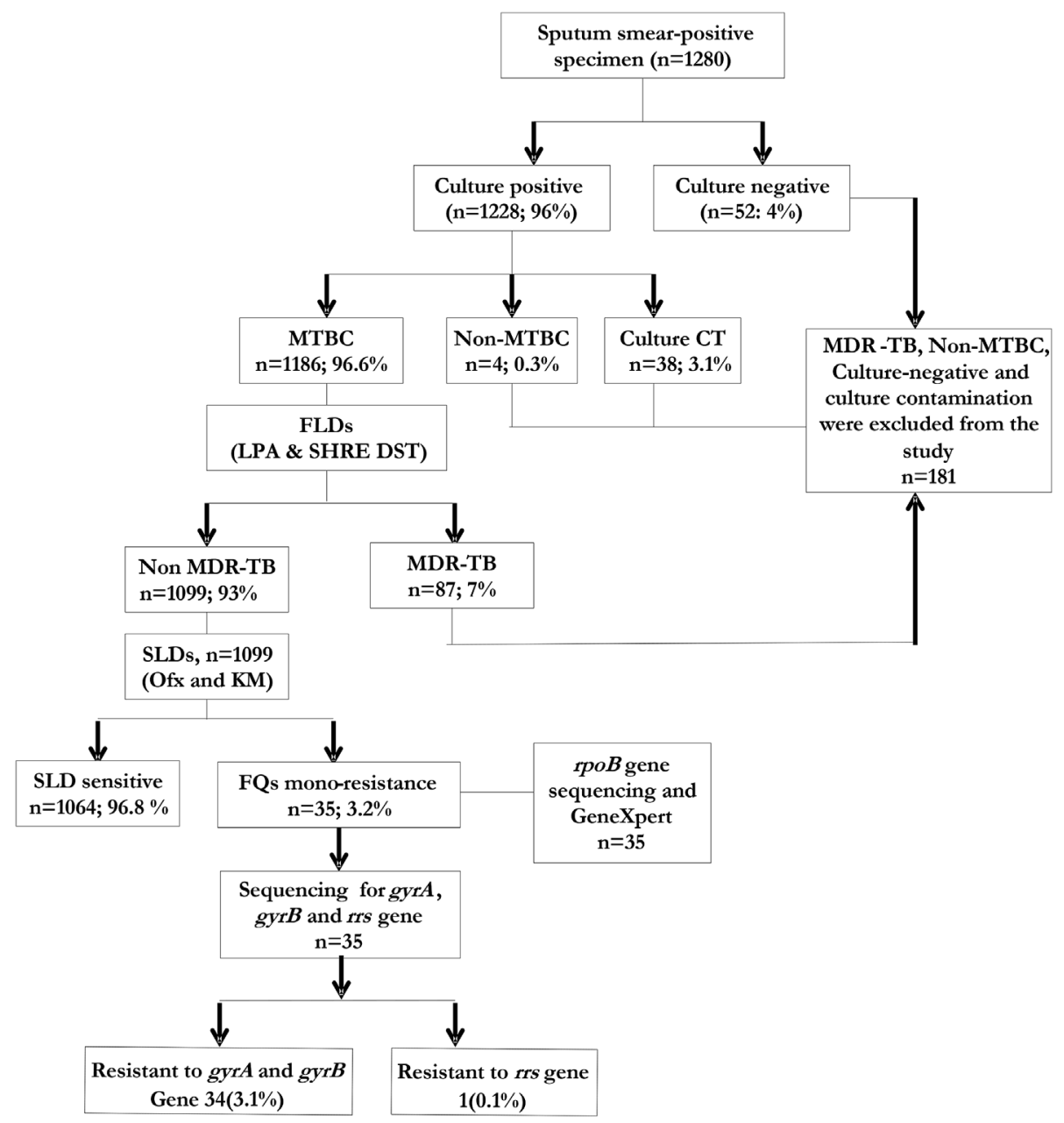

Fig. 1. Schematic diagram of work flow of the study.

Definition of abbreviation: LPA: line probe assay; MDR-TB: multidrug tuberculosis; R: rifampicin; H:isoniazid; S: Streptomycin; E; Ethambutol: DST: drug susceptibility testing; SLD: Second-line drugs; KM: kanamycin; Ofx:ofloxacin; Pre-XDR-TB: Pre-extensively drug-resistant TB; XDR-TB: extensively drug-resistant TB; FQ: Fluoroquinolones group of drugs, includes Ofx; Injectable groups of drugs includes KM; CT: contamination; MTBC: Mycobacterium tuberculosis complex.

Table 1

Primers used for PCR amplification and detection of drug-resistant M.tb.

\begin{tabular}{llll}
\hline Target gene & Primer sequence $\left(5^{\prime}-3^{\prime}\right)$ & $\begin{array}{l}\text { PCR } \\
\text { product } \\
\text { size }(\mathrm{bp})\end{array}$ & $\begin{array}{l}\text { Annealing } \\
\text { temp }\left({ }^{\circ} \mathrm{C}\right)\end{array}$ \\
\hline$r p o B$ & $\begin{array}{l}\text { CAGACGTTGATCAACATCCG } \\
\text { TACGGCGTTCGATGAAC }\end{array}$ & 305 & 56.7 \\
$g y r A$ & $\begin{array}{l}\text { CAGCTACATCGACTATGCGA } \\
\text { GGGCTTCGGTGTACCTCAT }\end{array}$ & 320 & 61.0 \\
gyrB & $\begin{array}{l}\text { CGCAAGTCCGAACTGTATGTCGTAG } \\
\text { GTTGTGCCAAAAACACATGC }\end{array}$ & 364 & 61.0 \\
& $\begin{array}{l}\text { AAGTACCCGCCTGGGGAGTACGG } \\
\text { GGTGGGACAACACCTGGAACAAGTC }\end{array}$ & 831 & 54.0 \\
\hline
\end{tabular}

$\mathrm{PCR}=$ Polymerase Chain Reaction .

early stage and treatment can be modified accordingly.

Xpert MTB/RIF provides the advantage of early and rapid diagnosis (turnaround time of $90 \mathrm{~min}$ as compared to eight weeks for solid culture, 42 days for liquid culture and $48 \mathrm{~h}$ for LPA) ${ }^{14}$. However, it is limited in its ability as it can only detect presence or absence of rifampicin resistance; on the other hand performance of LPA on smearpositives samples can give us additional information about INH resistance also but has slightly longer turnaround time. Recently, a study even evaluated the diagnostic performance of LPA among smear-negative pulmonary specimens and observed good sensitivity and
Table 2

Mutation pattern by DNA sequencing of gyrA, gyrB and rrs gene among drug sensitive isolates $(n=35)$.

\begin{tabular}{lllll}
\hline Gene & No. of strains (\%) & Nucleotide change & Types of mutation & DST results \\
\hline \multirow{2}{*}{ gyrA } & $06(17.14)$ & GCG-269-GTG & A 90 V & Resistant \\
& $09(25.71)$ & CGA-280-CTA & D 94 Y & Resistant \\
& $18(51.43)$ & GAC-281-GCC & D 94 A & Resistant \\
gyrB & $01(2.86)$ & AAC-1496-ACC & N 499 T & Resistant \\
$r r s$ & $01(2.86)$ & CAC-1401-CGC & A 1401 G & Resistant \\
\hline
\end{tabular}

Definition of abbreviation: alanine; D, aspartic acid; V, valine; E, glutamate; Q,glutamine; $\mathrm{Y}$, tyrosine; $\mathrm{N}$, asparagine; $\mathrm{T}$, threonine.

specificity. ${ }^{15}$ Conventional culture and DST has always been the gold standard for detecting $M t b$ with drug resistance patterns. However, culture sensitivity for detecting TB cases is highly benefited when clubbed with liquid culture (MGIT-960) techniques with an excellent sensitivity and specificity in high TB burden countries. ${ }^{16}$

The emergence of FQs resistance in recent years has been a cause of great concern and presently no precise data is available, especially among drug sensitive TB patients. In the present study, among FLD sensitive new TB cases the FQs mono-resistance was found to be $1.8 \%$ $(13 / 728)$ and among re-treated drug sensitive TB cases it was $5.9 \%$ (22/371). The overall FQ mono-resistance among drug sensitive TB cases including new and re-treated TB cases was 3.1\% (34/1099) and 
one $(0.1 \%)$ was found discordant in DNA sequencing. As per routine, DST using second-line drugs (FQs and aminoglycosides) is usually performed only for MDR-TB isolates. According to the WHO Tuberculosis report 2017, the proportion of MDR-TB cases with resistance to any fluoroquinolone (for which testing was done including ofloxacin, levofloxacin and moxifloxacin) was found to be $20 \%$ globally ${ }^{5}$ but there is paucity of data which can highlight the actual burden of FQs mono-resistant among drug sensitive TB cases.

The study findings revealed $100 \%$ concordance between Xpert MTB/RIF, LPA and DNA sequencing for $r p o B$ gene in the detecting of RIF resistance among first-line drug sensitive isolates (including those $35 \mathrm{Mtb}$ isolates which were FQ resistant) and all three techniques showed no RIF resistance in all 1099 cases.

Rising FQ resistance in India can be largely attributed to unregulated prescription of these drugs, their easy availability and use, as empirical anti-TB drugs by private practitioners. Studies have found development of resistance even with very short duration of treatment; resistance has been observed within 13 days of exposure to FQs, making many drug sensitive and treatment naïve patients prone for development of resistance. ${ }^{6}$ Some Indian researchers have reported about FQ resistance among MDR-TB cases. Jain et al., from Lucknow and Selvakumar et al. from Tamil Nadu have reported FQ mono-resistance among MDR-TB cases to the tune of $26 \%$ and $29 \%{ }^{4,7}$ respectively and a study of drug resistance surveillance (DRS), conducted by Ramachandran et al., in Gujarat in 2009, reported FQ mono-resistance to be around $24 \%$ with no significant difference between treatment naive and treatment-experienced TB cases. ${ }^{17}$ Few studies from India have hinted about emergence of FQ resistance among drug sensitive TB cases, but the literature is pretty scarce. A retrospective study at Antwerp, Belgium was conducted to evaluate the resistance for ofloxacin among new and retreated TB cases which showed ofloxacin mono resistance among new TB patients as $4.3 \%$ and among retreatment cases it was about $9.1 \%{ }^{18}$

Another study which was a one year FQs surveillance study conducted at Australia stated, that they found uncommon trend of FQ resistance among drug sensitive cases. ${ }^{19}$ The resistance to FQs remains uncommon in Australia as the availability for FQs is meticulously controlled by the government-subsidized medication scheme. ${ }^{20}$ In India presence of FQ resistance is a common problem among patients which needs to be addressed by the policy makers urgently so as to control the menace of DRTB in the coming years. Our study has found presence of FQ resistance (3.1\%) among FLD sensitive TB cases which further emphasizes the importance of using these drugs judiciously. Presently, the country is waiting eagerly for its first national drug resistance surveillance (NDRS) report, conducted by National Tuberculosis Institute (NTI) Bangalore, so that true resistance pattern among Indian patients along with regional variations can be estimated.

The present study's strength lies with the presence of a large sample size along with use of various diagnostic modalities including the conventional and newer molecular techniques for estimation resistance patterns and their confirmation. Sequencing of rrs gene gave information about one isolate $(2.8 \%)$ which had mutation at position 1401 (CAC-CGC). This present study had few limitations perhaps the biggest one is that in spite of generating useful data about presence of primary FQ mono-resistance among drug sensitive TB cases; a detailed follow up of such cases (35 patients) were not done which would have pragmatically captured the clinical implications.

In conclusion, the presence of FQ mono-resistance among drug sensitive TB cases can be a potential cause for treatment non-response or failure. Universal DST should be routinely performed in all sputum positive TB cases to evaluate the true spectrum and extent of drug resistance among them. Hence, Universal DST followed by an individualized regimen based on DST results seems like a perfect strategy to control TB in the country but this ideal dream has a host of logistic and economic nightmares attached to it.

\section{Funding}

Foundation for Innovative New Diagnostics, India and Department of Medicine, All India Institute of Medical Sciences, New Delhi, India.

\section{Conflicts of interest}

None of author has any conflict of interest.

\section{Ethics approval}

The study was approved by Ethics Committee of the All India Institute of Medical Sciences, New Delhi, India.

\section{Acknowledgments}

Authors acknowledge the help of Ms. Jigyasa Chaubey for managing and initial processing the specimens. We are also thankful to Mr. Sukhbir Singh for sample collection. Authors thanks to Central TB Division, Ministry of Health \& Family Welfare, Govt. of India; State TB Cell, National Capital Territory (NCT) of Delhi, Delhi. Foundation for Innovative New Diagnostics (FIND), India for logistic support. We are very thankful to the AIIMS administration for providing infrastructure and lab support.

\section{References}

1. World Health Organization. Guidelines for the programmatic management of drug-resistant tuberculosis. Emergency Update. . [accessed June 10, 2009]. Available from: http://whqlibdoc.who.int/publications/2008/9789241547581_eng.pdf; 2008.

2. Wang JY, Hsueh PR, Jan IS, et al. Empirical treatment with a fluoroquinolone delays the treatment for tuberculosis and is associated with a poor prognosis in endemic areas. Thorax. 2006:61:903-908.

3. Agrawal D, Udwadia ZF, Rodriguez C, Mehta A. Increasing incidence of fluoroquinolone resistant Mycobacterium tuberculosis in Mumbai, India. Int $J$ Tubercul Lung Dis. 2009;13:79-83.

4. Jain A, Dixit P, Prasad R. Pre-XDR \& XDR in MDR and Ofloxacin and Kanamycin resistance in non-MDR Mycobacterium tuberculosis isolates. Tuberculosis. 2012;92 404e406.

5. Global Tuberculosis Report. Geneva: World Health Organization; 2017.

6. Ginsburg AS, Woolwine SC, Hooper N, et al. The rapid development of fluoroquinolone resistance in M. Tuberculosis. N Engl J Med. 2003;349:1977-1978.

7. Selvakumar N, Kumar V, Balaji S, et al. High rates of ofloxacin resistance in Mycobacterium tuberculosis among both new and previously treated patients in Tamil Nadu, south India. PLoS One. 2015;10(3).

8. Siddiqi SH, Ruesch-Gerdes S. MGIT procedure Manual for BACTEC MGIT 960 TB System. Franklin Lakes, NJ: Becton Dickinson; 2006

9. Revised National Tuberculosis Control Programme Laboratory Network. Guidelines for quality assurance of smear microscopy for diagnosing tuberculosis. TBC India. Website. Available: http://www.tbcindia.nic.in/pdfs/RNTCP\%20Lab\%20Network\%20Guidelines; 2005.

10. World Health Organization. Policy statement. Molecular Line Probe Assay for Rapid Screening of Patients at Risk of Multidrug-resistant (MDR) TB. Geneva. 2008; 2008 Retrieved from: http://www.who.int/tb/features_archive/policy_statement.pdf.

11. Somerville W, Thibert L, Schwartzman K, Behr MA. Extraction of Mycobacterium tuberculosis DNA: a question of containment. J Clin Microbiol. 2005;43:2996-2997.

12. Williams DL, Waguespack C, Eisenach K, et al. Characterization of rifampin-resistance in pathogenic mycobacteria. Antimicrob Agents Chemother. 1994;38:2380-2386.

13. Jugheli L, Bzekalava N, de Rijk P, Fissette K, Portaels F, Rigouts L. High level of crossresistance between kanamycin, amikacin, and capreomycin among Mycobacterium tuberculosis isolates from Georgia and a close relation with mutations in the rrs gene. Antimicrob Agents Chemother. 2009;53:5064-5068.

14. World Health Organization. Rapid Implementation of the Xpert MTB/RIF Diagnostic Test: Technical and Operational 'how to' Practical Considerations. Geneva, Switzerland: WHO; 2011.

15. Singh BK, Sharma SK, Sharma R, et al. Diagnostic utility of a line probe assay for multidrug resistant-TB in smear-negative pulmonary tuberculosis. PLoS One. 2017;12(8):e0182988https://doi.org/10.1371/journal.pone.0182988.

16. World Health Organization. Use of Liquid TB Culture and Dug Susceptibility Testing (DST) in Low and Medium Income Setting. Summary Report of the Expert Group Meeting on the Use of Liquid Culture media. Geneva, Switzerland: WHO; 2007http://www.who.int/tb/ laboratory/use_of_liquid_tb_culture_summary_report.pdf.

17. Ramachandran R, Nalini S, Chandrasekar V, et al. Surveillance of drug-resistant tuberculosis in the state of Gujarat, India. Int J Tubercul Lung Dis. 2009;13(9):1154-1160.

18. Daniel O, Osman E, Bakare R, et al. Ofloxacin resistance among Mycobacterium Tuberculosis isolates in two states of south-west Nigeria. Afr J Respir Med. March 2011:18-20.

19. Ho J, Jelfs P, Sintchenko V. Fluoroquinolone resistance in non-multidrug-resistant tuberculosis- a surveillance study in New South Wales, Australia, and a review of global resistance rates. Int J Infect Dis. 2014;26:149-153.

20. Cheng AC, Turnidge J, Collignon P, Looke D, Barton M, Gottlieb T. Control of fluoroquinolone resistance through successful regulation, Australia. Emerg Infect Dis. 2012;18:1453-1460 